\title{
Vibroacoustic optimization of stiffening ribs and damping material distribution on sheet metal parts
}

\author{
M. Carfagni*, P. Citti, L. Governi and M. Pierini \\ Dipartimento di Meccanica e Tecnologie Industriali, Università degli Studi di Firenze, Italy
}

Ricordare Bruno Piombo è soprattutto pensare alla sua generosità e disponibilità all' amicizia. Con noi colleghi, molti dei quali gli erano amici, era sempre pronto alla battuta, a raccontare l'ultima barzelletta, ma anche a parlare con entusiasmo e senso critico di una mostra di pittura o di un libro letto. La sua compagnia arricchiva chi gli era accanto perchè oltre ad una elevata competenza professionale e ad una profonda preparazione scientifica Bruno rivelava una personalità poliedrica, dai mille interessi. Ciò nonostante il suo atteggiamento non era mai di superbia od ostentazione; sapeva porgersi agli altri con modestia e semplicità. La sua mancanza ci ha senza dubbio privato di un uomo colto e di un amico, ma il suo ricordo è ancora capace di trasmettere allegria ed amore per la vita.

\begin{abstract}
Vehicle noise and vibration levels are basic parameters in passenger comfort. Both static and dynamic stiffness of sheet metal parts is commonly increased by means of stiffening ribs. Vibrations are also reduced by adding a layer of damping material on the floor, the roof, the firewall and other parts of the vehicle. In common practice the panels to be treated are ribbed according to criteria based on the designer's experience, rather than on well defined design procedures and are uniformly covered by a layer of damping material. However, these are not efficient design solutions, especially with regard to the effectiveness of vibration reduction and to weight containment. In this paper a novel approach to achieve an optimal distribution of stiffening ribs and damping material will be presented. The proposed method is based on a Genetic Algorithm (G.A.) procedure which takes into account both the vibroacoustic performance and the weight and cost reduction. A simple case study will be illustrated to demonstrate the capabilities of the developed procedure.
\end{abstract}

\section{Introduction}

A method based on G.A. has been developed to optimize the design of dynamically excited panels to which stiffening ribs and damping material have been added to reduce vibrations and noise.

The two devices influence each other; in performing the optimization of a mechanical component where both ribs and damping material are to be used, the simultaneous effect of each of them should be taken into account. In this work, anyway, they have been considered separately in order to assess their effectiveness as standalone device.

Both the method and the results will be presented with reference to a simple case study to make them more readily understandable for the reader. As already mentioned, the developed optimization technique is based on the G.A. [1-5]. They operate according to a standard five-step procedure:

1. Encoding of the design variables. In this step, the values of the design variables representing the individual are set. The combinations of all the possible design variables form the algorithm's search space.

\footnotetext{
${ }^{*}$ Corresponding author. E-mail: monica.carfagni@unifi.it.
} 


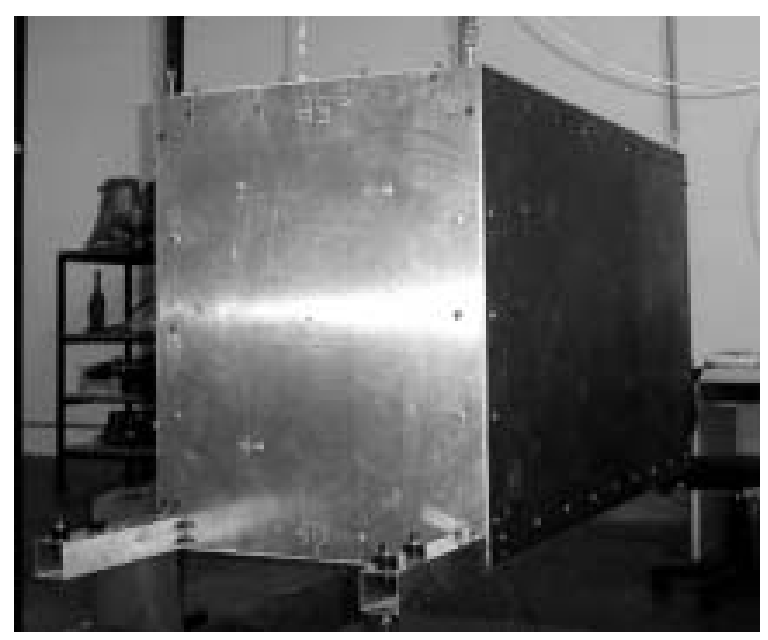

Fig. 1. The test structure ("box").

2. Computation of the objective function. In this step, each individual's performance is calculated.

3. Selection of the best-performing individuals. In this step, the best performing individuals are preserved and the worst performing ones are discarded according to a statistical criterion.

4. Crossover. In this step, the favorable individual's subparts are joined together to improve the whole [6-8].

5. Mutation. In this step, variability is introduced to prevent early algorithm convergence.

The G.A. has been implemented using Mathwork's Matlab to produce and manage the set of individuals making up the population, and using MSC/Patran and MSC/Nastran (respectively a Finite Element pre/post processor and a Finite Element solver) to model and analyze them.

\section{Definition and modeling of the test structure}

The modeling of the studied system, which is represented by a panel and alternatively a single layer of damping material or a set of stiffening ribs, has been devised according to the Finite Element Method (F.E.M.) principles.

The test structure is part of a simplified vehicle mock-up, named "box", made of an aluminum frame and 6 aluminum panels bolted onto it (Fig. 1).

In this work the box floor has been analyzed, a flat panel whose characteristics are:

- dimensions: $1548 \times 553 \mathrm{~mm}^{2}$;

- thickness: $3.1 \mathrm{~mm}$;

- Young's modulus (E): $7.35 \cdot 10^{10} \mathrm{~N} / \mathrm{m}^{2}$;

- density $(\rho): 3900 \mathrm{~kg} / \mathrm{m}^{3}$;

- Poisson ratio $(\nu)$ : 0.29 .

In Figs 2 and 3 are shown the F.E. models of the box floor used respectively for stiffening ribs optimization and for damping material optimization. The reasons for the differences between the model are provided below.

The stiffening ribs can be modeled by raising some of the nodes of the panel model above its plane, along a direction which is orthogonal to the plane itself. The raising length is a constant value specified by the designer at the beginning of the optimization procedure. While raising the nodes, each one has been considered to be independent from the others $[9,10]$. Accordingly, the total number of design variables $\mathrm{N}$ equals the number of nodes in the F.E. model. For the one shown in Fig. 2 the nodes belonging to the external frame (meshed using quadrilateral elements) cannot be raised, since they are interface nodes with the box structure.

Modeling the stiffening ribs by raising the nodes leads to a geometric ambiguity caused by four-sided elements. With all the node coordinates defined, the F.E. analysis provides correct, assuming sufficiently low deformation 


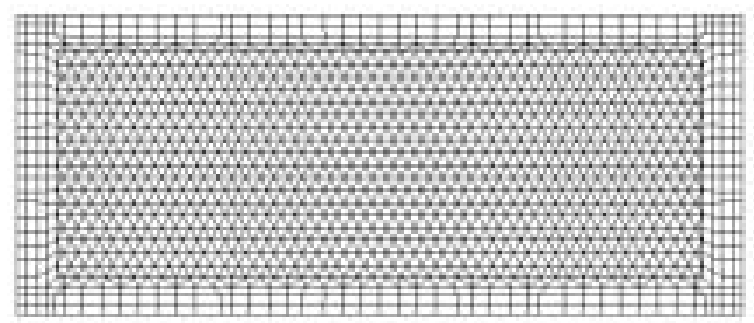

Fig. 2. Finite element model of the box floor for stiffening ribs optimization.

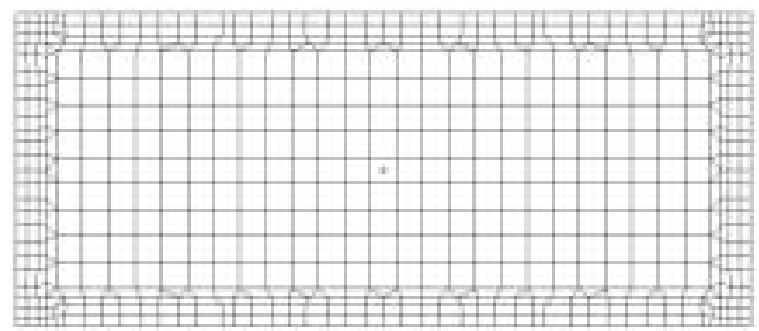

Fig. 3. Finite element model of the box floor for damping material optimization.
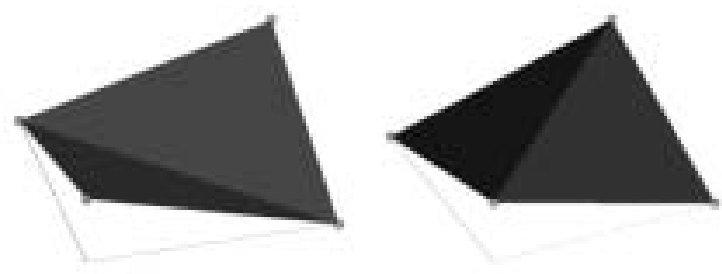

Fig. 4. Geometries associated to a square element with two opposite nodes raised.

of the element, and univocal results. Nevertheless, in the square element in Fig. 4 showing two diagonally raised nodes, the panel area is not univocally defined. We, therefore, used a mesh made of triangular elements (equilateral triangles, $20 \mathrm{~mm}$ per side) whose node coordinates served to univocally define the rib geometry.

The layer of damping material has been modeled by hexahedral solid elements with the following properties:

- thickness: $5 \mathrm{~mm}$;

- Young's modulus (E): $5 \cdot 10^{8} \mathrm{~N} / \mathrm{m}^{2}$;

- density $(\rho)=1700 \mathrm{~kg} / \mathrm{m}^{3}$;

- Poisson ratio $(\nu):=0.4$

- damping factor $(\eta)=0.4$.

The solid elements, representing the damping material patches, have been obtained by extruding the corresponding 4-noded plate elements. In this case the existence or the absence of the solid element represents a single design variable. Consequently the total number of design variables equals the number of four-sided elements of the inner region of the panel shown in Fig. 3.

It is clear that when dealing with the damping material distribution modeling, the ambiguity described above does not subsist any more, since there is no need to move the F.E. nodes from their original position. Accordingly, a regular quadrilateral element mesh was used.

Different element sizes have been compared keeping in mind the assumption that the maximum wave length in the frequency range of interest must be contained in 6 elements at least. In the case of Fig. 3, the final mesh exhibits quadrilateral elements with $54 \mathrm{~mm}$ side length. 
Moreover, several different dynamic analyses have been performed in order to find the best modeling strategy for the damping material:

- 1 layer of 8-noded solid elements;

- 2 layers of 8-noded solid elements;

- 1 layer of 20-noded solid elements;

- 2 layers of 20-noded solid elements.

The results have not shown significant differences in the frequency range $0 \div 200 \mathrm{~Hz}$, which was assumed to be our frequency range of interest. Accordingly, the selected solution was the first one because its calculation time was considerably shorter.

On the other hand, the side of the triangular elements used for the mesh shown in Fig. 2 is $20 \mathrm{~mm}$ long. The difference in the element basic dimension (smaller elements for rib modeling, larger elements for damping material modeling) is due to the necessity of a sufficiently accurate geometrical modeling of the ribbed region. On the other hand, the damping material patches can be larger, since a small damping material patch would be very hard to cut and to place on the panel.

In the G.A. the generic configuration of either stiffening ribs or damping material distribution is represented by a vector $\boldsymbol{v}$ (called individual) made of $N$ binary digits, where $N$ is the total number of design variables. If the $i^{\text {th }}$ element of $v$ is 0 , then:

- in the case of ribs optimization, the correspondent node in the model lays upon the panel's plane (original position). Conversely, if the value is 1 , the node is raised of a pre-defined amount $D_{z}$.

- in the case of damping material optimization, the correspondent damping material element in the model is "switched off"(i.e. the element does not exist in the model). Conversely, if the value is 1 , the element is "switched on".

According to this procedure the F.E. model of the flat panel is modified depending on the correspondent vector.

A population of the G.A. is made of a set of vectors $\boldsymbol{v}_{k}$ (i.e. a set of F.E. models obtained from the vectors). During the optimization procedure the initial population evolves, according to the G.A. rules, until a set of design variable values representing an optimal solution for the studied problem is obtained.

\section{Objective functions}

In order to evaluate the performance of a selected configuration of either stiffening ribs or damping material distribution, a proper Objective Function (O.F.) must be devised according to an estimate of the desired performance.

In this work two different O.F. formulations will be presented and discussed. Since the final objective is to minimize the panel's dynamic and acoustic responses, it has been decided to compute the O.F. for each individual on the basis of the node velocities, rather than on acceleration or displacement (velocity being directly proportional to sound pressure). The two O.F. formulations are respectively shown in Eqs (1) and (2).

$$
O F_{1}=\frac{1}{\Delta f \times N} \sum_{f=1}^{M} \sum_{i=1}^{N} \nu_{f i}
$$

Where:

- $f=$ frequency;

- $\Delta f=f_{\max }-f_{\min }=$ frequency range of interest $(0 \div 200 \mathrm{~Hz})$;

$-i=$ node;

- $M=$ number of spectral lines used in O.F. value computation;

$-N=$ number of nodes used in O.F. value computation;

$-\nu=$ node velocity modulus. 


$$
O F_{2}=A \times(1+K)
$$

$$
K=\frac{\bar{R}_{q}}{R_{q v}}
$$

$$
R_{q}=\sqrt{\frac{1}{\Delta f} \times \int_{f_{\min }}^{f_{\max }} y^{2} d f}
$$

Where:

- $A=$ area subtended by the average nodal velocity spectrum (defined later);

- $\Delta f=f_{\max }-f_{\min }=$ frequency range of interest $(0 \div 200 \mathrm{~Hz})$;

$-y=$ difference between velocity spectrum value, at each spectral line, and its mean value;

- $\bar{R}_{q}=R_{q}$ value for the analyzed individual;

- $R_{q v}=R_{q}$ value for the panel without any kind of treatment.

The first definition accounts for the average vibration level. For each spectral line, the velocities of all the nodes belonging to the panel are computed and the correspondent mean value is evaluated. By plotting such mean value versus the analysis frequency, the average nodal velocity spectrum is obtained (as the ones shown in Figs 9 and 11). The O.F. value is obtained by averaging the spectrum over the frequency range of interest.

The second definition is capable of taking into account both the mean spectrum value (factor A in Eq. (2)) and the spectrum shape, i.e. whether the spectrum is markedly undulated or not (factor $1+K$ in Eq. (2)).

By means of both the definitions, the performance of a given panel increases as the O.F. value decreases.

The G.A. - based procedure analyses the F.E. model corresponding to each individual $v_{k}$ in the frequency range of interest with a $1 \mathrm{~Hz}$ constant frequency step.

\section{The F.E. analysis}

The load condition acting on the box consists of two equal frequency-varying forces applied on the longitudinal frame beams (Fig. 5). Such forces, in their turn, cause a load distribution acting on the joint points between the floor panel and the box structure.

The vertical forces applied on the longitudinal frame beams were derived from an earlier experimental study, and so are assumed to represent a realistic excitation.

In order to perform a correct dynamic F.E. analysis, accounting for both the forces induced by the described load condition on the joint points and the inertia and stiffness effect of the box attached to the investigated panel, it is necessary to consider the F.E. model of the entire box. Nevertheless, such an analysis entails a much higher computation time than the one necessary to analyze the standalone floor. Considering that the proposed G.A. based procedure requires a large number of F.E. runs $(100 \div 300)$, the use of a complete box analysis proves to be an unfeasible solution.

To cope with this issue, the superelement technique was applied. By using this technique [11], only two reduced dimension matrixes (mass and stiffness) are necessary to simulate the entire box behavior; the two matrixes themselves make up the superelement of the structure.

The superelement technique is particularly effective because, with respect to the complete F.E. model of the box, it exhibits the following advantages:

- computation time is considerably reduced;

- memory usage is much smaller.

According to the superelement technique the entire box has been modeled as follows (Fig. 5):

- superelement of the box without the floor panel (Fig. 5 part a);

- floor panel (undergoing optimization) (Fig. 5 part b).

By using the described modeling procedure and the variables defined in Section 2, the G.A. on which the optimization procedure is based, underwent a series of preliminary test runs in order to allow the selection of the best set of the typical G.A. parameters $[2,4,6]$ 
Table 1

$O F_{1}$ values for three stiffening ribs configurations

\begin{tabular}{lc}
\hline Tested configurations & Objective function $O F_{1}$ \\
\hline Flat panel & $1.47 \cdot 10^{-3}$ \\
Panel with conventional stiffening ribs configuration & $1.34 \cdot 10^{-3}$ \\
Panel with optimized stiffening ribs & $1.21 \cdot 10^{-3}$ \\
\hline
\end{tabular}

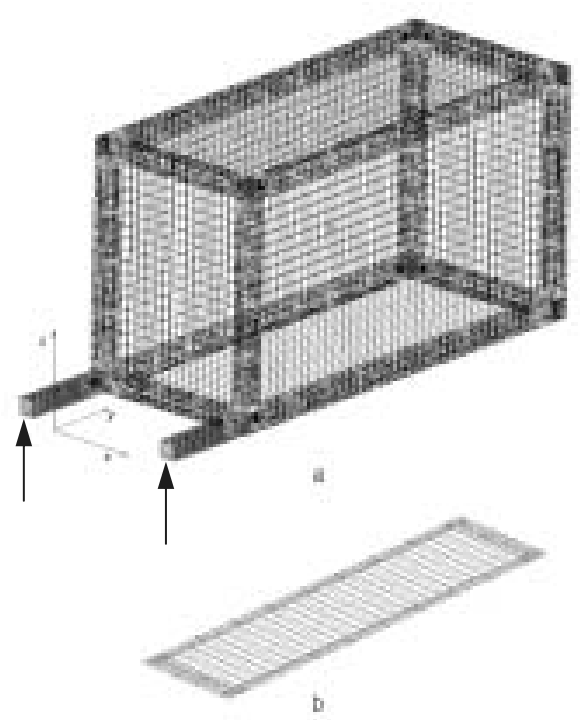

Fig. 5. Structure division according to the superelement technique. Floor-less box structure (a); panel undergoing optimization (b) The load forces are also shown.

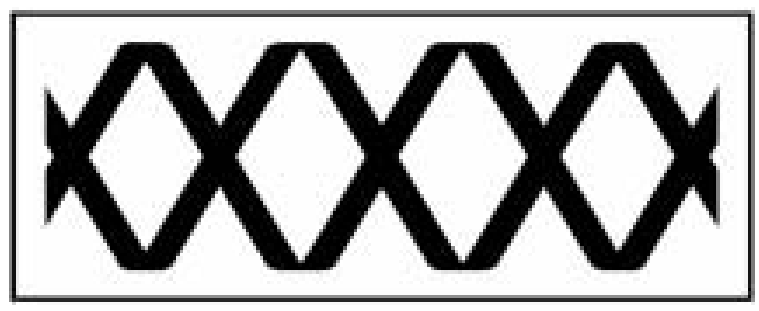

Fig. 6. Optimized panel's F.E. model.

\section{Results and discussion}

\subsection{Stiffening ribs optimization}

The O.F. shown in Eq. (1) $\left(O F_{1}\right)$ was tested with reference to the stiffening ribs optimization. The optimization was carried out using the proposed G.A. - based method. The result was compared to the performance, computed by means of Eq. (1), of the flat panel (with no stiffening ribs) and of a panel ribbed by a conventional configuration (called reference panel). The latter was selected among several conventional rib arrangements, since it proved to be the best performing.

Table 1 summarizes the $O F_{1}$ values of the three tested configurations. In Figs 6 and 7 the optimized panel and the reference one are shown respectively (in the figures, black areas indicate the presence of stiffening ribs, white areas lie on the panel's plane).

The results of Table 1 show that an evident improvement over the reference panel was achieved. Such improvement approximately equals the performance difference existing between the flat panel and the reference one. As a 
Table 2

$O F_{2}$ values for three damping material distributions

\begin{tabular}{lcc}
\hline Tested configurations & Objective function $O F_{2}$ & Damping material percentage \\
\hline Flat panel without damping material & $1.57 \cdot 10^{-1}$ & $0 \%$ \\
Panel with uniform damping material distribution & $6.58 \cdot 10^{-2}$ & $100 \%$ \\
Panel with optimized distribution of damping material & $5.26 \cdot 10^{-2}$ & $72 \%$ \\
\hline
\end{tabular}

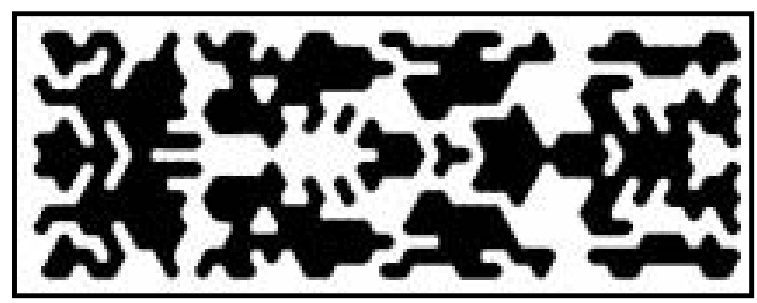

Fig. 7. reference panel F.E. model.

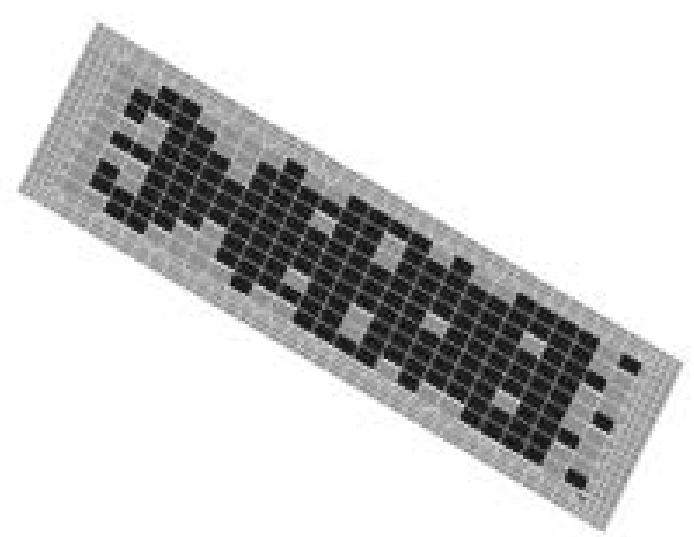

Fig. 8. Optimized panel's F.E. model.

consequence it is possible to assert that the use of the proposed optimization techniques proves to be very effective in the containment of the mean vibration level of the panel.

\subsection{Damping material distribution optimization}

The O.F. shown in Eq. (2) $\left(\mathrm{OF}_{2}\right)$ was tested with reference to damping material distribution optimization. The result obtained by the optimization method was compared to the performance, computed by means of Eq. (2), of the flat panel with no damping material and of a panel uniformly covered by a layer of damping material. The latter is the solution commonly exploited when dealing with the design of an actual industrial part.

Table 2 summarizes the $O F_{2}$ values of the three tested configurations. In Fig. 8 the optimized panel is shown. Since in $\mathrm{OF}_{2}$ formulation the spectrum shape plays a crucial role, in Fig. 9 the superposition of the average velocity spectra corresponding to the optimized panel and to the one with uniform damping material distribution is shown.

The results of Table 2 and the comparison of the two spectra plotted in Fig. 9 demonstrate a significant performance improvement achieved by means of the optimized configuration with regard to the uniformly treated panel. Moreover $28 \%$ of damping material is saved.

Examining the results obtained by the test carried out using $\mathrm{OF}_{2}$ formulation, it seemed to be useful to give the designer the opportunity to set the relative significance of the two terms in the trade-off between performance and 
Table 3

$O F_{2 c}$ values for three damping material distributions $(\alpha=0.8)$

\begin{tabular}{lcr}
\hline Tested configurations & Objective function $O F_{2 C}(\alpha=0.8)$ & Damping material percentage \\
\hline Flat panel without damping material & $1.57 \cdot 10^{-1}$ & $0 \%$ \\
Panel with uniform damping material distribution & $9.44 \cdot 10^{-2}$ & $100 \%$ \\
Panel with optimized distribution of damping material n & $9.32 \cdot 10^{-2}$ & $48 \%$ \\
\hline
\end{tabular}

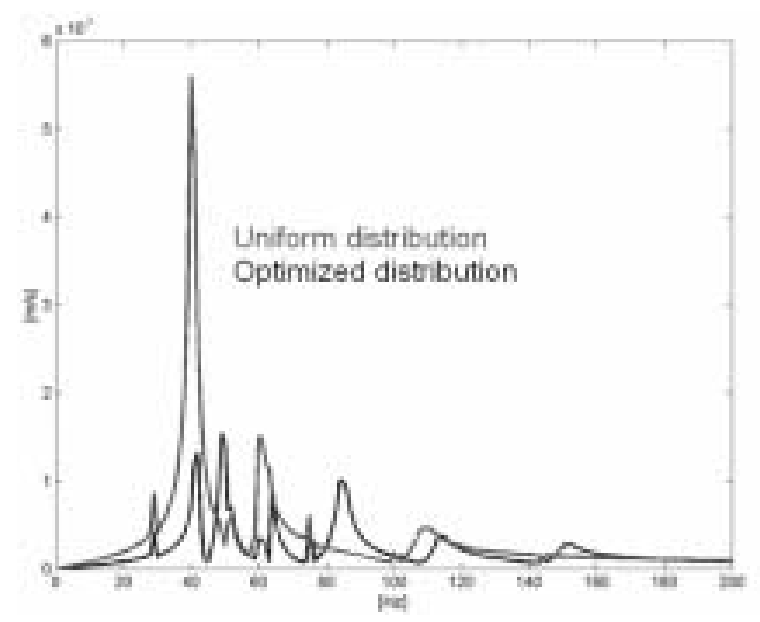

Fig. 9. Average velocity spectra.

cost. Accordingly, a modified version of $O F_{2}$, called $O F_{2 c}$, was devised as follows:

$$
O F_{2 C}=A \times(1+K)+\frac{O F_{2 \nu}-O F_{2 p}}{\bar{C}} \times \alpha \times C
$$

Where:

- $F_{2 \nu}=O F_{2}$ value for the flat panel with no damping material;

$-F_{2 p}=O F_{2}$ value for the flat panel with uniform damping material distribution;

$-C=$ number of damping material patches for the analyzed individual;

$-\bar{C}=$ number of damping material patches on the panel with uniform damping material distribution;

$-\alpha=$ cost coefficient.

The second addendum of Eq. (7) is a penalty factor increasing as the number of damping material patches increases. The selection of a proper value for the coefficient $\alpha(0 \leqslant \alpha \leqslant 1)$ allows to set the significance of the penalty factor on the overall value of the O.F.

In Table 3 the results obtained by the optimization method using the new O.F. $(\alpha=0.8)$ are summarized.

The optimized damping material configuration and the correspondent spectrum, compared with the one of the uniformly treated panel, are shown respectively in Figs 10 and 11.

The results of Table 3 and the two spectra of Fig. 11 prove that the proposed approach is very effective in reducing the total amount of damping material while preserving a very good dynamic behaviour (52\% of damping material is saved). The results demonstrate an O.F. value approximately equal between the optimized configuration and the one with uniform damping material distribution, though allowing only a very small amount of damping material to be used. The spectra, in their turn, confirm the achievement of the final goal.

\section{Conclusions}

A G.A. - based method for optimizing the arrangement of stiffening ribs and the distribution of damping material on dynamically excited panels has been illustrated. F.E. modeling was used to derive both rib arrangements and 


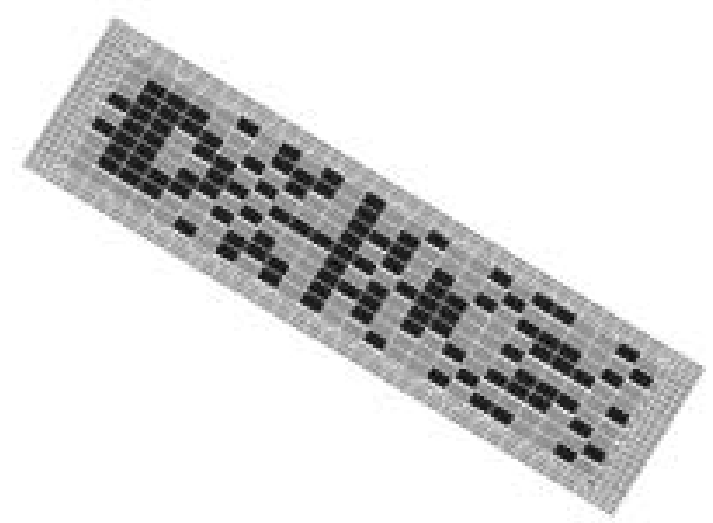

Fig. 10. Optimized panel's F.E. model.

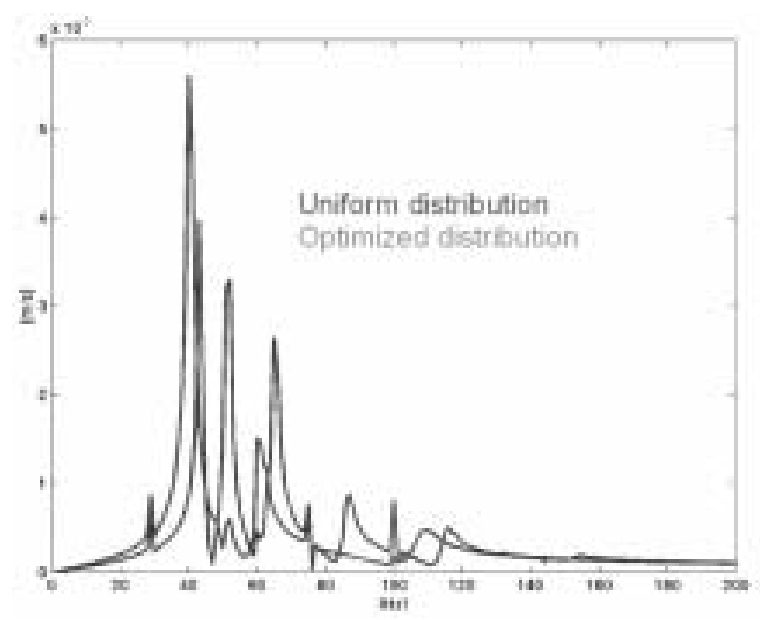

Fig. 11. Average velocity spectra.

damping material distribution. The ribs were modeled by raising some of the nodes of the tested panel model above its plane, each node position corresponds to a design variable. The damping material patches are modeled by solid elements attached to the plate elements making up the F.E. model of the test panel. Each patch corresponds to a design variable.

The proposed optimization method produces innovative rib arrangements and damping material distribution that are impossible to produce by conventional design techniques.

The developed method has been tested to the floor of a simplified vehicle mock-up. In order to correctly simulate the complete structure behavior and to speed up the procedure, the superelement technique has been used.

The results of a comparison of optimized panel dynamic performance and that of conventionally designed solutions validated the effectiveness of the former in reducing vibration levels. The results of damping material distribution optimization have shown that the method can be used either to reduce (up to 52\%) the damping material maintaining the same vibrational performance or to decrease the mean vibration level with the same amount of damping material. Forthcoming work will focus on increasingly complex mechanical assemblies, especially three-dimensional panel structures; in addition experimental testing will be performed in order to validate the F.E. results.

\section{Acknowledgments}

The authors are grateful to Centro Ricerche Fiat, Turin, Italy, for the provided support. 


\section{References}

[1] D. Goldberg, Genetic algorithms in search, optimization and machine learning, Addison Wesley, 1989.

[2] R.J. Povinelli, Improving computational performance of genetic algorithms: a comparison techniques, GECCO 2000 Late Breaking Papers, Las Vegas, NV, 2000.

[3] T. Blickle and L. Thiele, A comparison of selection schemes used in genetic algorithms, Technical Report 11, Computer Engineering and Communication Networks Lab (TIK), Zurich, CH, 1995.

[4] T. Blickle and L. Thiele, A mathematical analysis of tournament selection, Proceedings of ICGA95, San Francisco, CA, 1995.

[5] B.T. Zhang and J.J. Kim, Comparison of selection methods for evolutionary optimisation, Proceedings of Evolutionary Programming V, Cambridge, Massachusetts, 1995.

[6] K. Rasheed, Guided crossover: a new operator for genetic algorithm based optimisation, Technical Report HPCD-TR-50, Department of Computer Science, Rutgers University, New Brunswick, NJ, 1997.

[7] W.M. Spears and K.A. De Jong, On the virtues of parameterised uniform crossover, Proceedings of ICGA4, San Mateo, CA, 1991.

[8] W.M. Spears and V. Anand, A study of crossover operator in genetic programming, Proceedings of ISMIS 91, 1991.

[9] M. Carfagni, L. Governi and M. Pierini, A genetic algorithm method for optimizing rib arrangements in dynamically excited panels, ISMA 2000, Leuven, Belgium, 2000

[10] M. Carfagni, L. Governi and M. Pierini, Metodologia non convenzionale per la progettazione di piastre bugnate, AIAS Proceedings, Lucca, Italy, 2000.

[11] NAFEMS, A finite element primer, Italy, 1992. 

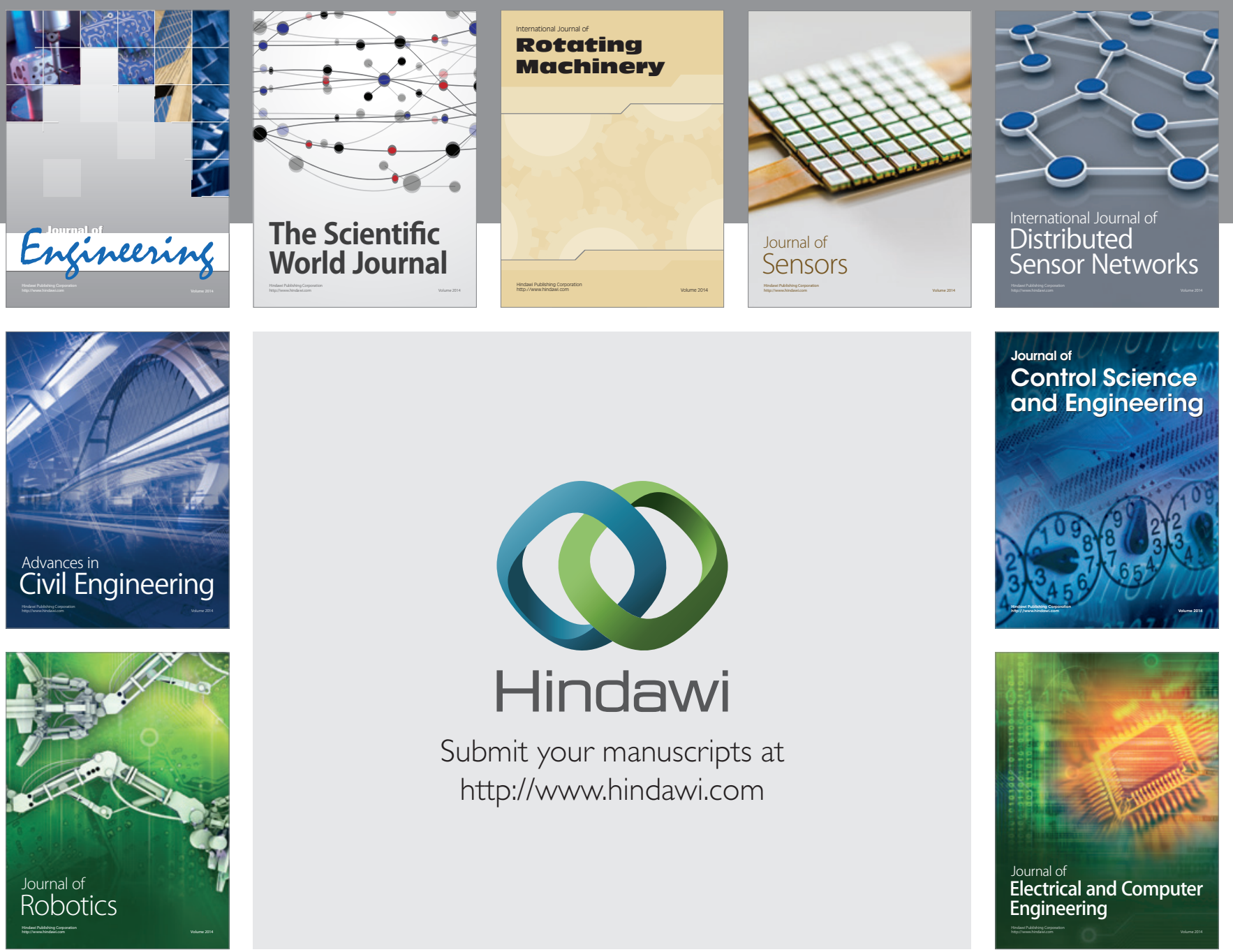

Submit your manuscripts at

http://www.hindawi.com
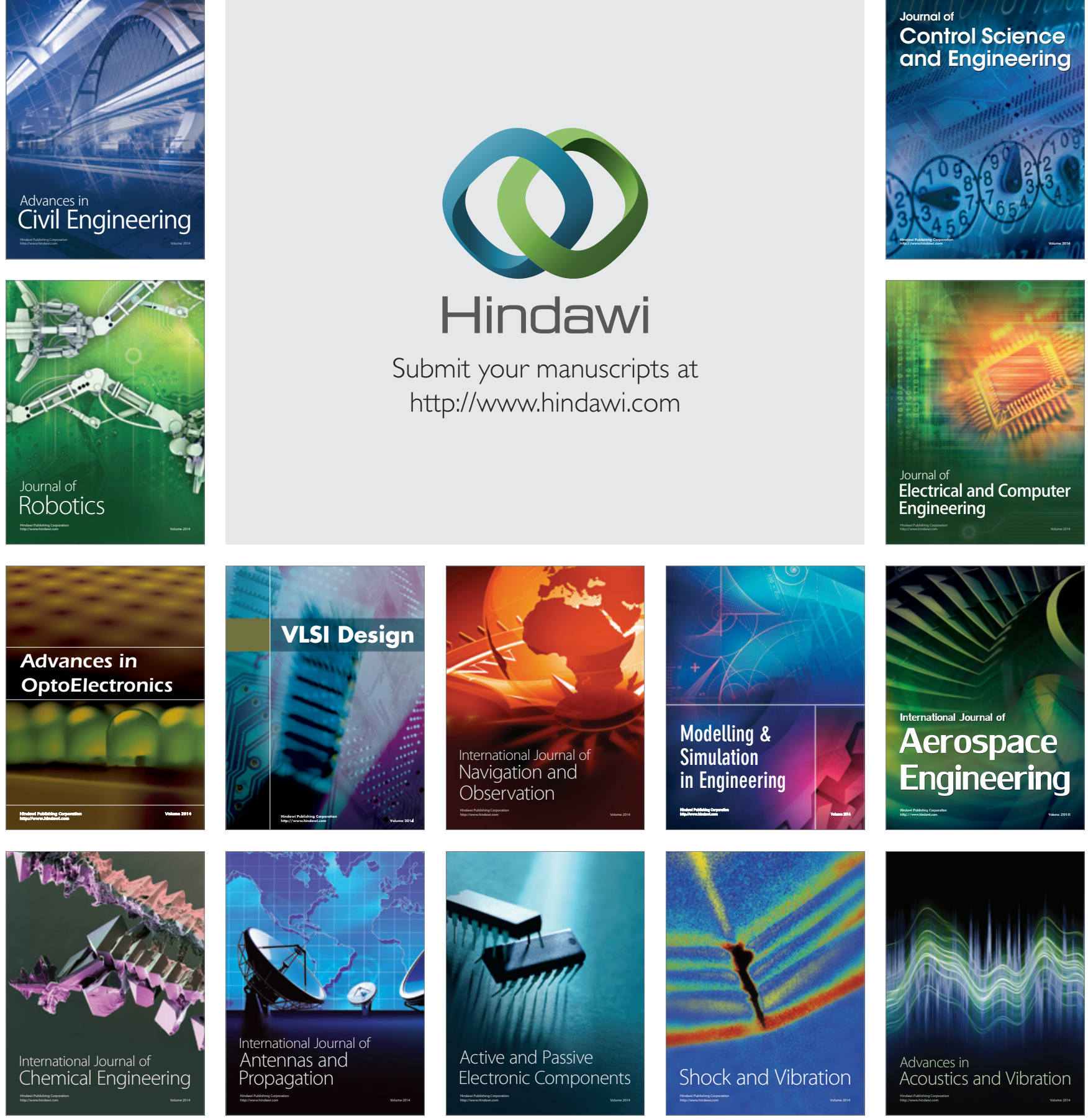